\title{
Standardization of the Fully Stapled Laparoscopic Roux-en-Y Gastric Bypass for Obesity Reduces Early Immediate Postoperative Morbidity and Mortality: A Single Center Study on 2606 Patients
}

\author{
Bruno Dillemans • Nasser Sakran • Sebastiaan Van Cauwenberge • Thibault Sablon • \\ Barbara Defoort • Els Van Dessel • Faki Akin • Nathalie Moreels • \\ Sebastiaan Lambert • Jan Mulier • Ravindra Date • Michel Vandelanotte • \\ Tom Feryn - Luc Proot
}

Received: 17 February 2009 / Accepted: 28 July 2009/Published online: 15 August 2009

(C) The Author(s) 2009. This article is published with open access at Springerlink.com

\begin{abstract}
Background Various techniques of laparoscopic Roux-en-Y gastric bypass have been described. We completely standardized this procedure to minimize its sometimes substantial morbidity and mortality. This study describes our experience with the standardized fully stapled laparoscopic Roux-en-Y gastric bypass (FS-LRYGB) and its influence on the 30-day morbidity and mortality.
\end{abstract}

B. Dillemans $\cdot$ S. Van Cauwenberge $\cdot$ T. Sablon $\cdot$ B. Defoort $\cdot$

E. Van Dessel $\cdot$ F. Akin $\cdot$ N. Moreels $\cdot$ S. Lambert $\cdot$

M. Vandelanotte $\cdot$ T. Feryn $\cdot$ L. Proot

Department of General Surgery, AZ Sint-Jan Hospital AV,

Bruges, Belgium

N. Sakran

Department of General Surgery A, Hillel Yaffe Medical Center, Hadera, Israel

\section{J. Mulier}

Department of Anaesthesia, AZ Sint-Jan Hospital AV, Bruges, Belgium

R. Date

Department of Gastrointestinal Surgery,

Lancashire Teaching Hospital NHS Foundation Trust,

Lancashire, UK

B. Dillemans $(\bowtie)$

Department of General Surgery, AZ Sint-Jan Hospital AV Brugge,

Ruddershove 10,

8000 Bruges, Belgium

e-mail: bruno.dillemans@azbrugge.be
Methods We retrospectively analyzed 2,645 patients who underwent FS-LRYGB from May 2004 to August 2008. Operative time, hospital stay and readmission, re-operation, and 30-day morbidity/mortality rates were then calculated. The 30-day follow-up data were complete for 2,606 patients $(98.5 \%)$.

Results There were 539 male and 2,067 female patients. Mean age was 39.2 years (range 14-73), mean BMI $41.44 \mathrm{~kg} / \mathrm{m}^{2}$ (range, 23-75.5). The mean hospital stay was 3.35 days (range 2-71). Mean total operative time was 63 min (range 35-150).

One patient died of pneumonia within 30 days of surgery $(0.04 \%)$. One hundred and fifty one $(5.8 \%)$ patients had postoperative complications as follows: gastrointestinal hemorrhage $(n=89,3.42 \%)$, intestinal obstruction $(n=9$, $0.35 \%)$, anastomotic leak $(n=5,0.19 \%)$ and others $(n=47$, $1.80 \%)$. In 66 patients, the bleeding resolved without any surgical re-intervention. One hemorrhage resulted in hypovolemic shock with subsequent renal and hepatic failure.

Conclusion The systematic approach and the full standardization of the FS-LRYGB procedure contribute highly to the very low mortality and the low morbidity rates in our institution. Gastrointestinal bleeding appears to be the commonest complication, but is self-limiting in the majority of cases. Our approach also significantly reduces operative time and turns the technically demanding laparoscopic Roux-en-Y gastric bypass procedure into an easy reproducible operation, effective for training.

Keywords Morbid obesity · Bariatric surgery $\cdot$ Roux-en-Y gastric bypass $\cdot$ Laparoscopy $\cdot$ Complications 


\section{Introduction}

Obesity is a chronic disease and is associated with an increased risk of co-morbidities and mortality. The World Health Organization has declared obesity as the epidemic of the twenty-first century. An estimated 300 million people worldwide are obese [1]. More than $65 \%$ of Americans are overweight. Of these, $30 \%$ are obese and $4.9 \%$ are extremely obese [2].

In Europe, the prevalence of obesity (body mass index $\geq 30 \mathrm{~kg} / \mathrm{m}^{2}$ ) in men range from $4.0 \%$ to $28.3 \%$ and in women from $6.2 \%$ to $36.5 \%$ [3].

In 1991, a consensus was reached by the National Institutes of Health stating that surgical treatment offers the best long-term results [4]. Bariatric surgery is effective in reducing obesity related co-morbidities as well as achieving major long-term weight loss and improvement in quality of life. Furthermore, loss of excess weight leads to an almost normal life expectancy $[5,6]$. Compared with conservative management, bariatric surgery leads to a $29 \%$ reduction in the long-term risk of death [7].

Bariatric operations are classified as either restrictive or malabsorptive. Roux-en-Y gastric bypass (RYGB) combines both mechanisms. The first gastric bypass was reported in 1967 by Mason and Ito [8]. Wittgrove and Clark were the first to report the laparoscopic procedure in 1994 [9]. The RYGB has steadily gained increasing popularity and is now the most common bariatric operation worldwide [10]. The laparoscopic approach has a clear advantage over the traditional open one including better visualization of the operative field, decreased pain, lower incidence of wound-related and abdominal wall complications, better cosmetic results, faster recovery, and shorter hospital stay. The 5-year weight-loss rate and the resolution of co-morbidities are similar [11, 12].

The Department of General Surgery in AZ Sint-Jan Hospital AV in Bruges is a referral center with a specific unit dedicated to the treatment of morbid obesity. Approximately 1,000 obese patients are treated each year. The objective of this study was to evaluate the impact of the total standardization and the full stapling of the laparoscopic RYGB procedure on the 30-day morbidity and mortality.

\section{Materials and Methods}

This is a retrospective study of a prospectively kept database of all the patients undergoing fully stapled laparoscopic Roux-en-Y gastric bypass (FS-LRYGB) in a standardized fashion by a single surgeon or under his direct supervision, from May 3rd 2004 to August 31st 2008.

Patient data were collected from the hospital's electronic medical record and by telephone interview. The data included patient demographics, body mass index, operative times, length of stay, technical details, and 30-day readmission, re-operation, morbidity, and mortality rates.

Data are expressed as mean for continuous variables or as percentage for categorical data, and were analyzed using Excel 2007 for Windows XP.

Operation Technique

\section{Anesthesia}

No premedication was given. One dose of cefazoline $1 \mathrm{~g}$ was given IV at induction. General anesthesia was induced with IV sufentanil and propofol, followed by IV cisatracurium to facilitate endotracheal tube placement and pneumoperitoneum inflation. General anesthesia was maintained with incremental IV bolus of sufentanil, cisatracurium, and inhalation of desflurane as required. Standard physiologic monitoring included electrocardiograph, pulse oximetry, and non-invasive arterial blood pressure.

All the patients were ventilated mechanically at a $50 \%$ inspired oxygen fraction $\left(\mathrm{FIO}_{2}\right)$ and a positive endexpiratory pressure around $5 \mathrm{cmH}_{2} \mathrm{O}$, using a volume or a pressure controlled setting. Patients were ventilated at an elevated $\mathrm{EtCO}_{2}$ level to improve blood pressure and skin perfusion [13, 14].

\section{Patient Positioning and Trocar Placement}

The patient is placed in the supine position, split-leg with reverse Trendelenburg position. All patients get a flexion of the hips to help increase surgical abdominal workspace [15]. The surgeon stands between the legs. A video monitor is positioned at the level of the patient's head. A $30^{\circ}$ angle scope is used. Abdominal insufflation with carbon dioxide $\left(\mathrm{CO}_{2}\right)$ is achieved using a Veress needle. Intra-abdominal pressures are maintained at $15 \mathrm{mmHg}$.

A five-port technique is employed: a $10 \mathrm{~mm}$ port 10 $15 \mathrm{~cm}$ below the xiphoid process, a $5 \mathrm{~mm}$ port high epigastric on the midline, a 12-mm port in the right upper quadrant and two $12 \mathrm{~mm}$ ports in the left upper quadrant. The latter two ports are placed on the same line of the $10 \mathrm{~mm}$ port, while the former $12 \mathrm{~mm}$ port is placed somewhat higher above the same line (sub costal) (Fig. 1).

\section{Creation of the Gastric Pouch}

A small window is made between the lesser omentum and the lesser curvature of the stomach, entering the lesser sac 5-6 cm below the gastro-esophageal junction (Fig. 2a). At this point a linear stapler (EndoGia Universal Stapler System, Covidien, USA or Echelon 60 Endopath Stapler, Ethicon, USA), usually with a blue cartridge, is introduced through this window and the stomach horizontally cut over 
Fig. 1 Schematic overview of the trocar positioning and the different trocar sizes. An overview of the different instruments that are used by each trocar is depicted in the most right column

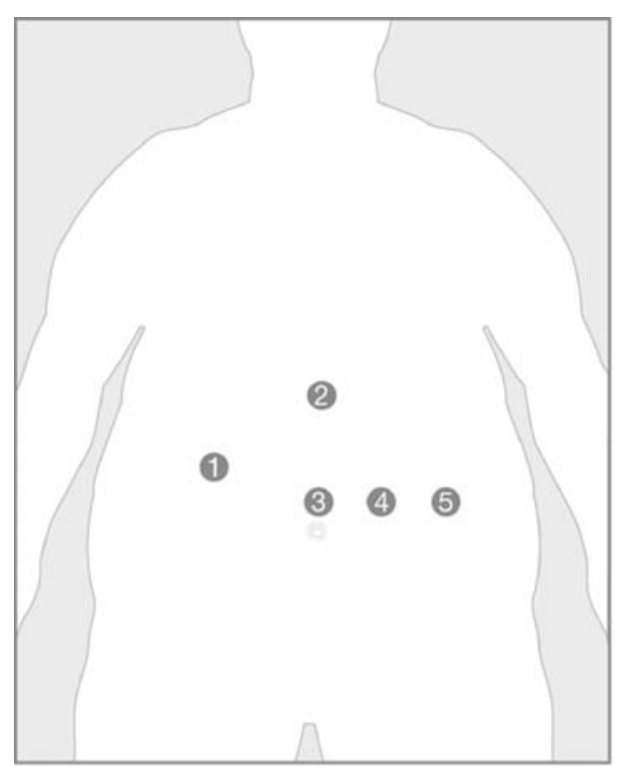

a distance of $50 \mathrm{~mm}$ (Fig. 2b). A second linear 60-mm stapler is then introduced and fired to vertically transect the stomach using a $34 \mathrm{Fr}$. orogastric tube as a guide (Fig. 2c). The next step consists of dissection and opening of the angle of His. Posterior to the stomach the dissection is completed to create a window at the angle of His. Finally, the pouch is completed by vertically firing one or two more $60 \mathrm{~mm}$ cartridges in the direction of and through the latter created window, along the gastric tube (Fig. 2d).

\section{Creation of the Gastro-jejunostomy}

A small opening is made in the lower left corner of the pouch, the opening stretched and a purse-string suture is sewn using an absorbable monofilament suture (PDS II 3/0, Ethicon, USA; Fig. 3a-b). A 25-mm Premium Plus CEEA (Covidien, USA) is then introduced intra-abdominally via the most left lateral, manually-dilated trocar opening. The anvil is brought into the gastric pouch opening and the pursestring tied (Fig. 3c). Thereafter, the greater omentum is lifted and divided up to the left side of the transverse colon. Next, the ligament of Treitz is identified and a loop of jejunum is antecolically pulled up from this point to the gastric pouch (Fig. 3d). An enterotomy is created $30-50 \mathrm{~cm}$ from Treitz and the Premium Plus CEEA introduced over 5-6 $\mathrm{cm}$ in the jejunal loop via this opening in the distal direction. We then perforate the jejunum under slight traction with the spike, remove the spike, and connect the stapler to the anvil (Fig. 4a-c). The anastomosis is completed by closing and firing the instrument. The remaining small bowel loop with the previously created opening is then transected $1 \mathrm{~cm}$ proximal to the gastro-jejunostomy using a linear stapler with a $60-\mathrm{mm}$ white cartridge so as to avoid a long blind loop of jejunum (Fig. 4d).

\section{Creation of the Jejuno-jejunostomy}

The length of the alimentary limb is standard $130 \mathrm{~cm}$. In patients with BMI $>50 \mathrm{~kg} / \mathrm{m}^{2}, 200 \mathrm{~cm}$ is measured and an antimesenteric opening is created both in the alimentary and the biliopancreatic limb. A linear $60 \mathrm{~mm}$ stapler with a white cartridge is introduced in both openings in order to establish a side-to-side anastomosis (Fig. 5a-b). The resulting enterotomy defect is lifted by three holding stitches ((PDS II 4/0, Ethicon, USA) and then longitudinally closed using a similar stapler (Fig. 5c). The final step is transecting the remaining blind loop of the biliopancreatic limb, also with a similar linear stapler, and removal of the piece of bowel (Fig. 5d).

\section{Testing of the Gastro-jejunostomy}

A leakage test is done by forcefully injecting methylene blue through the orogastric tube at the level of the gastrojejunostomy anastomosis (Fig. 6). The gastro-jejunostomy is reinforced if necessary with some additional stitches of an absorbable monofilament suture (PDS II 4/0, Ethicon, USA). To prevent postoperative bleeding, all staple lines are inspected under an elevated systolic arterial pressure above $140 \mathrm{mmHg}$ [16].

The left lateral trocar port site, which was enlarged to introduce the Premium Plus CEEA, is closed with the help of the Endo Close trocar site closure device (Covidien, USA) to prevent 'lateral entrapment' or herniation.

\section{Postoperative Care}

On the first postoperative day patients are kept nil by mouth. No routine upper GI imaging series are performed. Oral intake is restarted on the second postoperative day and 
Fig. 2 Creation of the gastric pouch. a Start of the dissection at the lesser curvature 5-6 cm below the gastro-esophageal junction. b The first linear stapler cuts the stomach horizontally. c Vertical transection of the stomach along a 34 Fr. orogastric tube. d Final stapling at the angle of His
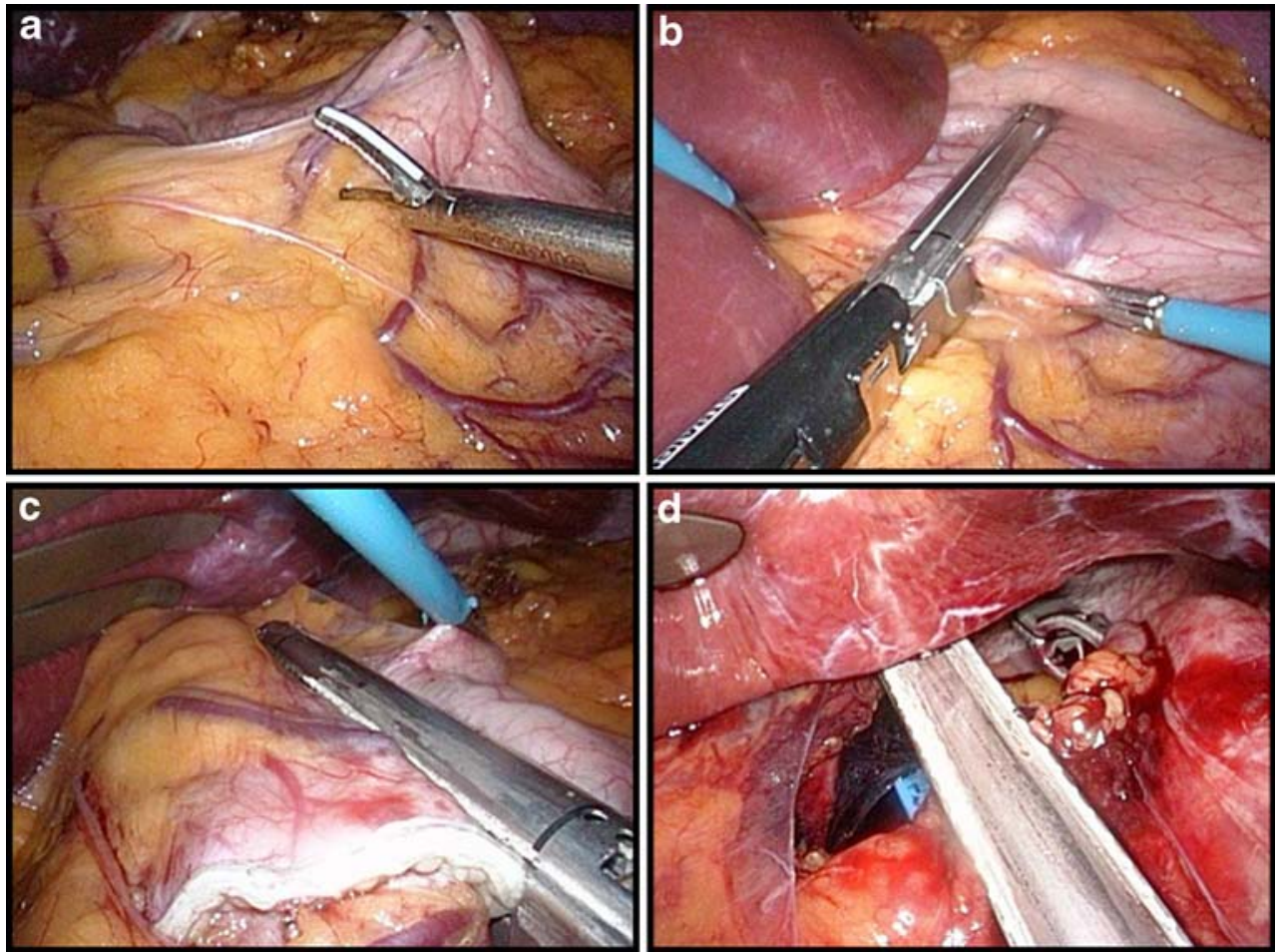

the patients are discharged on average on the third postoperative day with specific dietary instructions. To prevent deep venous thrombosis patients receive a daily subcutaneous injection with low-molecular-weight heparins for 14 days postoperatively together with elastic compres- sion stockings. In addition, a proton pump inhibitor (omeprazole $20 \mathrm{mg}$ ) is started and continued for 3 months to prevent marginal ulcer formation. The first follow-up visit is scheduled for after 6 weeks. Thereafter, visits are planned 6,12 , and 24 months postoperatively.
Fig. 3 Creation of the gastrojejunostomy (part 1). a Opening of the gastric pouch in the lower left corner. b Purse-string suturing with PDS 3/0. c Introduction of the anvil of the circular stapler and tying of the pursestring. d Identification of the angle of Treitz
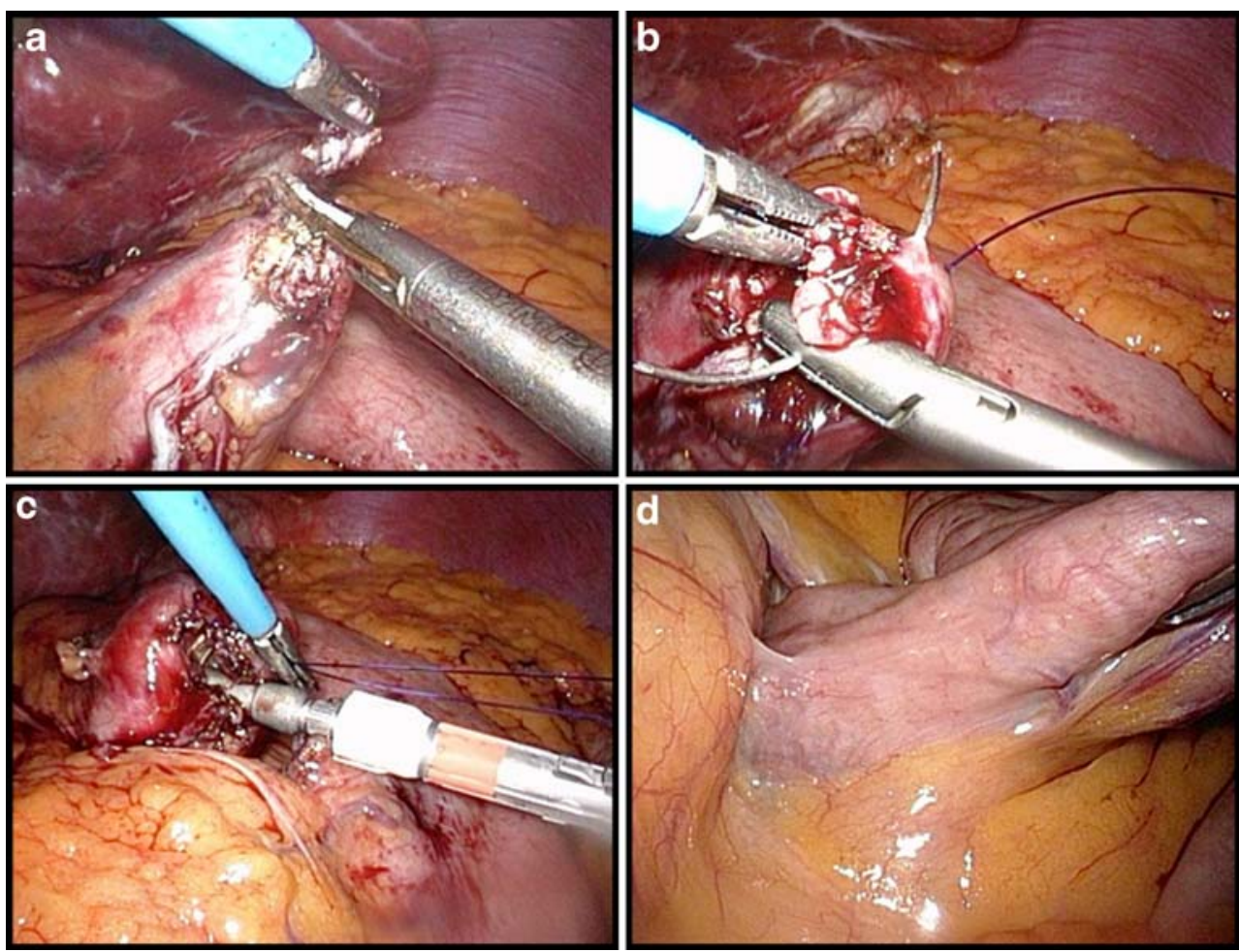
Fig. 4 Creation of the gastrojejunostomy (part 2). a Enterotomy $30-50 \mathrm{~cm}$ from the angle of Treitz. b Antimesenteric perforation of the jejunum with the spike after introduction of the circular stapler. c Closure of the circular stapler. d Finalization of the anastomosis by transection of the remaining small bowel $1 \mathrm{~cm}$ proximal to the gastro-jejunostomy

Fig. 5 Creation of the jejunojejunostomy. a Antimesenteric opening in the alimentary limb. b Side-to-side anastomosis with a linear $60 \mathrm{~mm}$ stapler. c Closure of the enterotomy defect using three stay sutures. d Transection of the remaining blind loop of the biliopancreatic limb
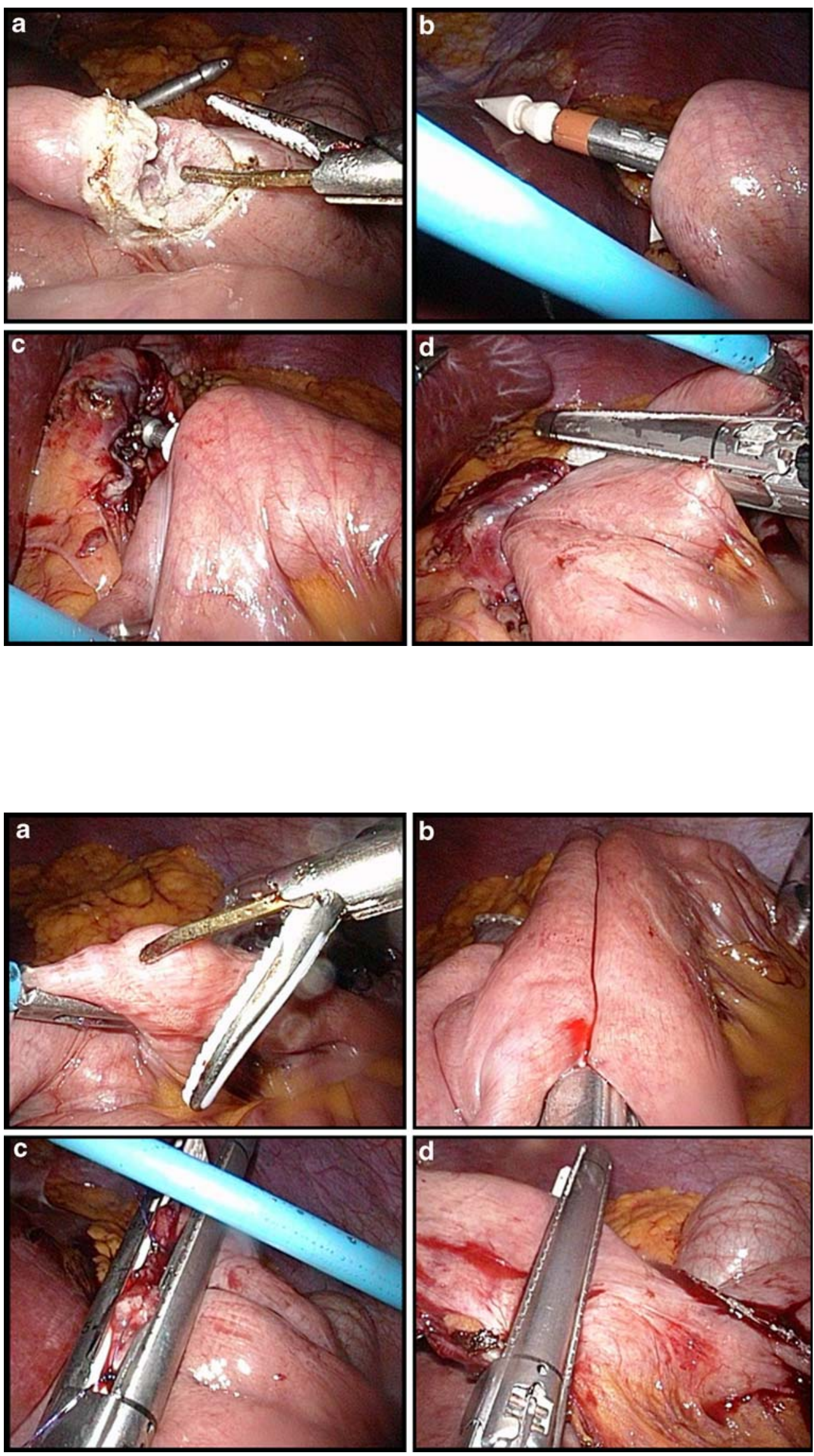


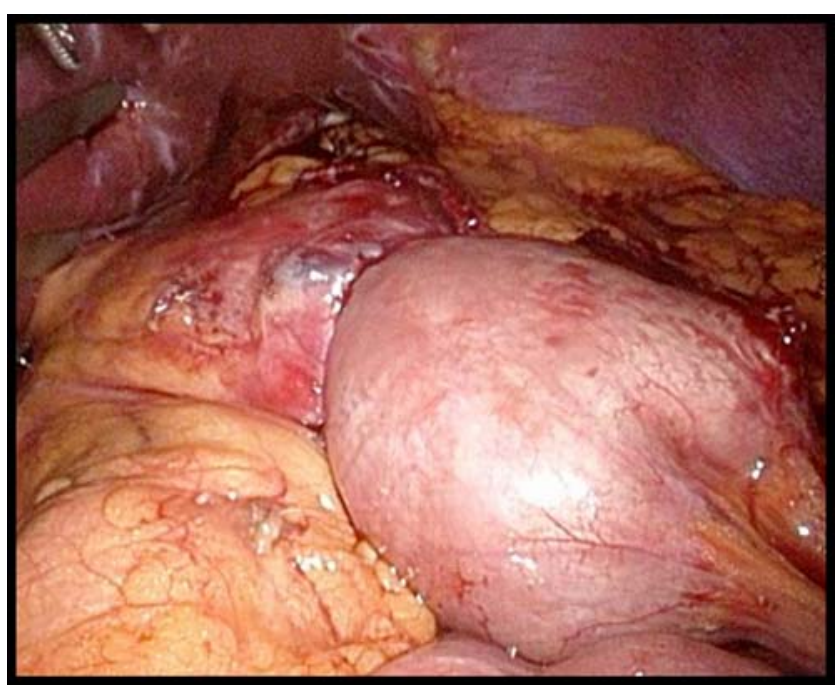

Fig. 6 Testing of the gastro-jejunostomy with methylene blue

\section{Results}

Surgical Volume and Patient Characteristics

Between May 2004 and August 2008, 4,732 bariatric procedures were performed in AZ Sint-Jan Hospital AV Bruges. Of these, 2,645 were FS-LRYGB, 190 were open Roux-en-Y gastric bypasses (RYGB), 798 were laparoscopic vertical banded gastroplasties (VBG, MacLean), 1,078 were laparoscopic adjustable gastric bandings (LAGB) and 21 laparoscopic sleeve gastrectomies (LSG).

In-hospital data were collected for all patients. The 30day follow-up data were complete for 2606 patients (98.5\%) of the FS-LRYGB group.

The number of FS-LRYGB procedures performed doubled between $2005(n=459)$ to $2007(n=847)$. The female to male ratio was $4: 1$. The mean age was 39.2 years (14-73) with $81.3 \%$ of patients younger than 50 years of age and $3.2 \%$ of patients older than 60 years. The mean BMI was $41.44 \mathrm{~kg} / \mathrm{m}^{2}$ with a range from 23 to $75.5 \mathrm{~kg} / \mathrm{m}^{2}$. Three hundred sixty patients $(13.81 \%)$ were super-obese $\left(\mathrm{BMI} \geq 50 \mathrm{~kg} / \mathrm{m}^{2}\right)$ and a total of 53 patients $(2.03 \%)$ had a $\mathrm{BMI} \leq 30$. The latter group $(\mathrm{BMI} \leq 30)$ were all patients that underwent a conversion from a previous restrictive procedure to a FS-LRYGB. The majority of the patients were Belgian; the center also has a high referral from the United Kingdom and the Netherlands (Table 1).

\section{Comorbid Diseases and Previous Surgery}

Pre-operative co-morbidities included hypertension (745 cases, 28.59\%), gastro-esophageal reflux disease (GERD; 715 cases, 27.44\%), dyslipidemia (617 cases, 23.68\%), diabetes mellitus type 2 (239 cases, 9.17\%), and sleep apnea (102 cases, 3.91\%) (Table 1).
FS-LRYGB was the primary procedure in 2,353 patients (90.29\%); the remaining underwent a conversion from a previous restrictive procedure. One hundred eighty-five patients had previous LAGB, 67 had a VBG and one patient had a LSG. Overall, 769 patients (29.5\%) had previously undergone non-bariatric abdominal surgery (other abdominal and/or pelvic operations).

\section{Length of Surgery and Hospital Stay}

Mean operative time was $63 \mathrm{~min}(35-150)$ and has importantly decreased in time as the team got more familiarized with the different standardized steps of the procedure. The mean length of hospital stay was 3.35 days. Of the 2,606 patients, 2,197 (84.31\%) were discharged on the third postoperative day or earlier.

No conversions to an open procedure occurred during the primary procedure. Synchronous procedures included cholecystectomy in 153 (5.87\%), abdominal wall hernia repair in $13(0.50 \%)$ and Nissen fundoplication in 9 $(0.35 \%)$ cases.

In-hospital and 30-day Morbidity Rate (Table 2)

Hemorrhage was the most common complication after FSLRYGB (89 patients, 3.42\%). Early bleeding was recognized by tachycardia, blood pressure drop, hematocrit drop, oliguria, drain output (in case of extra-luminal bleeding), hematemesis, and melena (in case of intra-luminal bleeding).

Table 1 General patient Characteristics $(n=2,606)$

\begin{tabular}{ll}
\hline Patient Characteristics & Variable \\
\hline Demographics & \\
Mean Age, years & 39.2 \\
$\geq 60$ & 84 patients \\
Sex, F:M & $4: 1$ \\
Mean body mass index (BMI) & 41.44 \\
Country of origin & \\
Belgium & $1602(61.47 \%)$ patients \\
Great Britain & $358(13.74 \%)$ \\
Netherlands & $335(12.85 \%)$ \\
Denmark & $175(6.72 \%)$ \\
Norway & $35(1.34 \%)$ \\
Other countries & $101(3.88 \%)$ \\
Co-morbidities & \\
Hypertension & $745(28.59 \%)$ \\
GERD & $715(27.44 \%)$ \\
Hypercholesterolemia & $617(23.68 \%)$ \\
Diabetes type 2 & $239(9.17 \%)$ \\
Sleep apnea & $102(3.91 \%)$ \\
\hline
\end{tabular}


Table 2 Incidence of early major postoperative complications ( $\leq 30$ days)

\begin{tabular}{lr}
\hline Complications & No. of cases (\%) \\
\hline Death & $1(0.04 \%)$ \\
Hemorrhage & $89(3.42 \%)$ \\
Blood transfusion & $46(1.77 \%)$ \\
Intestinal obstruction & $9(0.35 \%)$ \\
Pneumonia & $6(0.23 \%)$ \\
Pulmonary emboli and DVT & $5(0.19 \%)$ \\
Anastomotic leak & $5(0.19 \%)$ \\
Anastomotic stenosis and food impaction & $4(0.15 \%)$ \\
Pancreatic injury & $2(0.08 \%)$ \\
Small bowel perforation & $1(0.04 \%)$ \\
Intra-abdominal abscess & $1(0.04 \%)$ \\
\hline
\end{tabular}

In 66 patients, the bleeding resolved without any surgical reintervention. Of these patients, 46 required transfusions of blood products and fluids, while 20 others were managed without blood transfusion. In 23 patients, a laparoscopic reoperation was necessary to control bleeding. Of these 23 patients, a distinct bleeding source was found and controlled in 18 patients. In nine patients, the bleeding was extraluminal and in six patients intra-luminal; in the former group, at a clearly defined spot at the linear gastric staple line in all cases; in the latter, at the circular anastomotic staple line construction in all but one. Three patients had a bleeding from the trocar site or the mesentery of the small bowel. In five patients, no clear source of bleeding was found during laparoscopy and cleansing of the abdomen with clot removal stopped the bleeding. One of these patients later developed a subphrenic abscess which required re-operation, drainage, and antibiotic treatment. One hemorrhage resulted in hypovolemic shock with subsequent multi-organ failure (hepato-renal and pulmonary failure) and a total hospital stay of 71 days.

A leak developed in five patients $(0.19 \%)$ despite a negative intra-operative methylene blue leakage test. All but one were male patients. Four leaks were identified in the early postoperative phase (postoperative day $2(n=2)$ and day 3 $(n=2))$. One leak was discovered on the 26th day postoperatively. Patients were re-operated either laparoscopically $(n=4)$ or open $(n=1)$. All leaks were anastomotic: four at the gastro-jejunostomy and one at the jejuno-jejunostomy. No leaks at the other staple lines were observed.

Small bowel obstruction occurred in nine patients $(0.35 \%)$. Seven were secondary to trocar-site hernia ('lateral entrapment'). One patient suffered from an incarcerated umbilical hernia. One other patient had an obstruction at the level of the jejuno-jejunostomy due to an acute anastomotic angulation.
Other complications occurred in 47 patients (1.80\%). Two male patients had an iatrogenic pancreatic injury, which resulted in pancreatitis and was managed by laparoscopic drainage of the lesser sac.

One male patient developed an iatrogenic small bowel perforation which was controlled by primary closure of the perforation.

Other complications included wound infection in 12 $(0.46 \%)$, fever of unknown origin in nine $(0.35 \%)$, pneumonia in six $(0.23 \%)$, urinary tract infection in six $(0.23 \%)$, DVT/pulmonary embolism in five $(0.19 \%)$, early stenosis in four $(0.15 \%)$, and cardiac arrhythmia in two $(0.08 \%)$ cases.

The overall number of re-interventions was 49 on a total of 41 patients with a resulting re-operation rate of $1.57 \%$. Five re-operations were performed in patients already discharged from the hospital. Other 30-day re-admissions not leading to a re-intervention occurred in 29 patients. This accounts for a readmission rate of $1.30 \%$.

\section{In-hospital and 30-day Mortality Rate}

One patient (male, BMI $51 \mathrm{~kg} / \mathrm{m}^{2}$ ) died due to pneumonia and subsequent heart failure on the fourth postoperative day. This accounts for a $0.04 \%$ overall in-hospital and 30day mortality rate.

\section{Discussion}

The laparoscopic Roux-en-Y gastric bypass is considered as the golden standard among the surgical bariatric options. Despite the growing worldwide spread and surgical experience of this procedure, LRYGB remains a technically challenging procedure with a substantial morbidity and mortality.

Buchwald et al. reported in a large meta-analysis a mortality rate of $0.16 \%$ for LRYGB [17]. The mortality rate in our series was $0.04 \%$ which is among the lowest reported incidences in literature. Flum et al concluded that advancing age, male sex, and lower surgeon volume are associated with a higher risk of early death after bariatric surgery [18]. Although the mean BMI of our patient population is rather low compared to other series, we are convinced that besides the high patient volume, the full stapling and extensive standardization of the procedure are the main reasons for these favorable results. In this standardization, the procedure is split into different phases in which every participant (surgeon, registrar, scrub nurse, anesthesiologist) has a specific role. Every single laparoscopic maneuver has been completely rationalized which contributed to a substantial shortening of the length of the operative time. One must fear postoperative leaks as the most important cause of surgical related mortality. Gastric or intestinal leaks can 
result in severe peritonitis, sepsis, and multi-organ failure. Male gender, re-operation, older age, a BMI $>50 \mathrm{~kg} / \mathrm{m}^{2}$ and surgeon experience are all associated with poor operative outcome and a greater leakage rate $[19,20]$. The most common site for an anastomotic leak is the gastro-jejunostomy [20].

The leakage rate in our series was $0.19 \%(n=5)$. One patient underwent open re-operation and the other four patients were treated laparoscopically. Different studies report an overall leakage rate ranging from $0.1 \%$ to $5.25 \%$ [21-31] (Table 3). In a review of 6,135 patients in 13 selected series of laparoscopic RYGB, the mean leakage rate was $1.4 \%$ (range 0-4.3) [20]. The low leakage rate in this study is in our opinion attributed to a standardized anastomotic technique with suture reinforcement if necessary and the construction of a rather long gastric tube. The anastomotic integrity of the gastro-jejunostomy is intra-operatively assessed by high-pressure testing of this anastomosis with methylene blue. The methylene blue test has been reported to be $100 \%$ sensitive and to have no side-effects [32].

We prefer to use the circular stapler to complete the gastro-jejunostomy. Compared to the linear technique, this technique does not require closure of a common opening, eliminating a possible leakage site. Furthermore, traction on the gastro-jejunostomy is limited due to an equal distribution of forces on the anastomosis. Additionally, the circular stapler with a fixed diameter reproduces the opening of the anastomosis in each case without the subjective sizing of the other techniques [22].

Insertion of the circular stapler ( $25 \mathrm{~mm}$ Premium Plus CEEA) with the anvil trans-abdominally is easy, safe and quick. Passing the anvil into the gastric pouch trans-orally is an alternative but surgeons should be aware of possible technical difficulties as hypopharyngeal or esophageal injury has been described [33, 34].

The technique of the FS-LRYGB in our center involves the construction of a rather long $(5-6 \mathrm{~cm})$ but narrow pouch. A longer pouch facilitates the construction of the gastro- jejunostomy importantly and decreases the traction on it, especially when the alimentary limb is pulled up antecolically. Capella et al. are convinced that long narrow pouches are the most effective operations in bariatric surgery. Long narrow pouches have less tendency to enlarge and delay the transit of food to a greater degree than wider pouches, according to the Laplace's and Poiseuille's Laws. However, pouches are not perfect cylinders; the walls are not rigid and are of variable distensibility. Strict application of these physical laws to the clinical setting will require further research [35].

Hemorrhage was the most common in-hospital complication $(3.42 \%)$ occurring after FS-LRYGB in our experience. This number is consistent with the reported 3.1\% incidence by Spaw et al. [36] in a literature review of 2,895 patients. The most common sites of bleeding are the staple lines which are likely to bleed either extra-luminally (intraabdominally) or intra-luminally. At this point, additional care is taken to identify and control bleedings intraoperatively. Early recognition of postoperative bleeding is crucial. Careful clinical and hemodynamic monitoring of the patient in combination with the observation of the output of the intra-abdominal drain can avoid hypovolemic shock. In this study, $74.16 \%$ of postoperative bleedings were managed conservatively without the need of a surgical re-intervention. More recently, endoscopic management has been introduced to control bleeding from the gastro-jejunal staple line.

Early stenosis still remains an important issue. Of the 2,606 patients, $4(0.15 \%)$ were re-admitted within 30 days because of vomiting due to a narrowing of the gastro-jejunal anastomosis. All cases were treated endoscopically with balloon dilatation. However, stenosis becomes more apparent after the 30-day period. The overall stenosis rate in our series with a completed follow-up of 3 months was $0.95 \%$ ( $n=24$ out of 2,521 patients; follow-up rate of $95.3 \%$ ).

Small bowel obstruction occurred in nine patients $(0.35 \%)$ and nearly all were related to hernias. Seven out of nine patients had a lateral entrapment at the left lateral trocar
Table 3 Comparative overall leakage rate after Roux-en-Y gastric bypass

\begin{tabular}{lcc}
\hline 1st Author, year & No. of cases & Overall anastomotic leakage rate (\%) \\
\hline Carrasquilla [22], 2004 & 1,000 & 0.1 \\
Agaba [31], 2008 & 1,364 & 0.15 \\
Durak [27], 2008 & 1,133 & 1.5 \\
Gonzalez [26], 2007 & 3,018 & 2.1 \\
Madan [23], 2006 & 300 & 3.0 \\
Lee [24], 2007 & 3,828 & 3.9 \\
Hamilton [25], 2003 & 210 & 4.3 \\
Ballesta [30], 2008 & 1,200 & 4.9 \\
DeMaria [28], 2002 & 281 & 5.1 \\
Marshall [21], 2003 & 400 & 5.25 \\
Carucci [29], 2006 & 904 & 5.3 \\
\hline
\end{tabular}


site and required re-operation. This problem has now been overcome by using standard direct visual closure of the trocar site with the Endo Close (Covidien, USA) system.

In the past, we did not close Petersen's space. Although the antecolic antegastric technique has the least incidence of internal hernias, we have, as of March 2008, started closing Petersen's space. To date, there is sufficient data suggesting that this closure is better done on a routine basis [37-39]. However, recently, Madan et al. [40] suggest that mandatory closure of mesenteric defects might potentially create an increased risk of complications and costs without a real added benefit for the patient.

In this study, the re-operation rate was $1.57 \%$ and the readmission rate $1.30 \%$.

In other publications, readmission rates after gastric bypass range from $0.6 \%$ to $6.6 \%$ [41-44]. A nationwide inpatient sample estimated that the rates of unexpected reoperation during the same admission ranged from 6 to $9 \%$ [45].

\section{Conclusion}

The fully stapled laparoscopic Roux-en-Y gastric bypass (FS-LRYGB) is a safe and easy reproducible surgical weight-loss procedure. Maximal standardization of the operation and high surgical volume contributed both to the very low 30-day morbidity and mortality rate in our series. Postoperative bleeding was the commonest complication that in most cases could be treated conservatively. Further refinements in the stapling devices are mandatory to decrease this incidence in the future.

Open Access This article is distributed under the terms of the Creative Commons Attribution Noncommercial License which permits any noncommercial use, distribution, and reproduction in any medium, provided the original author(s) and source are credited.

\section{References}

1. Haslam DW, James WP. Obesity. Lancet. 2005;366(9492):1197209.

2. Hedley AA, Ogden CL, Johnson CL, et al. Prevalence of overweight and obesity among US children, adolescents, and adults, 1999-2002. JAMA. 2004;291(23):2847-50.

3. Berghofer A, Pischon T, Reinhold T, et al. Obesity prevalence from a European perspective: a systematic review. BMC Public Health. 2008;8:200.

4. Gastrointestinal surgery for severe obesity. Consens Statement $1991 ; 9(1): 1-20$.

5. van Gemert WG, Adang EM, Greve JW, et al. Quality of life assessment of morbidly obese patients: effect of weight-reducing surgery. Am J Clin Nutr. 1998;67(2):197-201.

6. Karlsson J, Sjostrom L, Sullivan M. Swedish obese subjects (SOS)-an intervention study of obesity. Two-year follow-up of health-related quality of life (HRQL) and eating behavior after gastric surgery for severe obesity. Int J Obes Relat Metab Disord. 1998;22(2):113-26.

7. Sjostrom L, Narbro K, Sjostrom CD, et al. Effects of bariatric surgery on mortality in Swedish obese subjects. N Engl J Med. 2007;357(8):741-52.

8. Mason EE, Ito C. Gastric bypass in obesity. Surg Clin North Am. 1967;47(6):1345-51.

9. Wittgrove AC, Clark GW, Tremblay LJ. Laparoscopic gastric bypass, Roux-en-Y: preliminary report of five cases. Obes Surg. 1994;4(4):353-357.

10. Buchwald H, Williams SE. Bariatric surgery worldwide 2003. Obes Surg. 2004;14(9):1157-64.

11. Wittgrove AC, Clark GW. Laparoscopic gastric bypass, Roux-enY- 500 patients: technique and results, with 3-60 month followup. Obes Surg. 2000;10(3):233-9.

12. Lujan JA, Frutos MD, Hernandez Q, et al. Laparoscopic versus open gastric bypass in the treatment of morbid obesity: a randomized prospective study. Ann Surg. 2004;239(4):433-7.

13. Mulier JP, Sels A, Dillemans B. Use of pressure support ventilation during laparoscopic bariatric surgery. Eur J Anesthesiology. 2008;25(S44):78.

14. Mulier JP, Dillemans B, Feryn T, et al. Adominal physiology and its impact on laparoscopic bariatric surgery. Obes Surg. 2008; 18:443.

15. Mulier JP, Dillemans B, Luyten A, et al. Horizontal positioning of the trunk and maximal leg flexion increases the laparoscopic workspace for bariatric surgery. Obes Surg. 2008;18:442.

16. Mulier JP, Dillemans B, Vandrogenbroeck G, et al. The effect of systolic arterial pressure in bleeding of the gastric stapling during laparoscopic gastric bypass surgery. Obes Surg. 2007;17:1051.

17. Buchwald H, Estok R, Fahrbach K, et al. Trends in mortality in bariatric surgery: a systematic review and meta-analysis. Surgery. 2007;142(4):621-32.

18. Flum DR, Salem L, Elrod JA, et al. Early mortality among Medicare beneficiaries undergoing bariatric surgical procedures. JAMA. 2005;294(15):1903-8.

19. Nguyen NT, Rivers R, Wolfe BM. Factors associated with operative outcomes in laparoscopic gastric bypass. J Am Coll Surg. 2003;197(4):548-55. discussion 555-7.

20. Gonzalez R, Nelson LG, Gallagher SF, et al. Anastomotic leaks after laparoscopic gastric bypass. Obes Surg. 2004;14(10):1299-307.

21. Marshall JS, Srivastava A, Gupta SK, et al. Roux-en-Y gastric bypass leak complications. Arch Surg. 2003;138(5):520-3. discussion 523-4.

22. Carrasquilla C, English WJ, Esposito P, et al. Total stapled, total intra-abdominal (TSTI) laparoscopic Roux-en-Y gastric bypass: one leak in 1000 cases. Obes Surg. 2004;14(5):613-7.

23. Madan AK, Lanier B, Tichansky DS. Laparoscopic repair of gastrointestinal leaks after laparoscopic gastric bypass. Am Surg. 2006;72(7):586-90. discussion 590-1.

24. Lee S, Carmody B, Wolfe L, et al. Effect of location and speed of diagnosis on anastomotic leak outcomes in 3828 gastric bypass cases. J Gastrointest Surg. 2007;11(6):708-13.

25. Hamilton EC, Sims TL, Hamilton TT, et al. Clinical predictors of leak after laparoscopic Roux-en-Y gastric bypass for morbid obesity. Surg Endosc. 2003;17(5):679-84.

26. Gonzalez R, Sarr MG, Smith CD, et al. Diagnosis and contemporary management of anastomotic leaks after gastric bypass for obesity. J Am Coll Surg. 2007;204(1):47-55.

27. Durak E, Inabnet WB, Schrope B, et al. Incidence and management of enteric leaks after gastric bypass for morbid obesity during a 10year period. Surg Obes Relat Dis. 2008;4(3):389-93.

28. DeMaria EJ, Sugerman HJ, Kellum JM, et al. Results of 281 consecutive total laparoscopic Roux-en-Y gastric bypasses to treat morbid obesity. Ann Surg. 2002;235(5):640-5. discussion 645-7. 
29. Carucci LR, Turner MA, Conklin RC, et al. Roux-en-Y gastric bypass surgery for morbid obesity: evaluation of postoperative extraluminal leaks with upper gastrointestinal series. Radiology. 2006;238(1):119-27.

30. Ballesta C, Berindoague R, Cabrera M, et al. Management of anastomotic leaks after laparoscopic Roux-en-Y gastric bypass. Obes Surg. 2008;18(6):623-30.

31. Agaba EA, Shamseddeen H, Gentles CV, et al. Laparoscopic vs open gastric bypass in the management of morbid obesity: a 7year retrospective study of 1, 364 patients from a single center. Obes Surg. 2008;18(11):1359-63.

32. Christian DJ, Barba C, Burke-Martindale C, et al. A simple bedside evaluation to detect gastroesophageal leaks after gastric bypass. Surg Endosc. 2004;18:S193. (abst).

33. Nguyen NT, Wolfe BM. Hypopharyngeal perforation during laparoscopic Roux-en-Y gastric bypass. Obes Surg. 2000;10(1):64-7.

34. Ikramuddin S, Ramathan RC, Gourash W, et al. Laparoscopic Roux-en-Y gastric bypass using a stapled EEA vs GIA technique for the gastrojejunal anastomosis. Obes Surg. 2000;10:132.

35. Capella RF, Iannace VA, Capella JF. An analysis of gastric pouch anatomy in bariatric surgery. Obes Surg. 2008;18(7):782-90.

36. Spaw AT, Husted JD. Bleeding after laparoscopic gastric bypass: case report and literature review. Surg Obes Relat Dis. 2005;1(2):99-103.

37. Higa K, Boone K, Arteaga Gonzalez I, et al. Mesenteric closure in laparoscopic gastric bypass: surgical technique and literature review. Cir Esp. 2007;82(2):77-88.
38. Cho M, Pinto D, Carrodeguas L, et al. Frequency and management of internal hernias after laparoscopic antecolic antegastric Roux-en-Y gastric bypass without division of the small bowel mesentery or closure of mesenteric defects: review of 1400 consecutive cases. Surg Obes Relat Dis. 2006;2(2):87-91.

39. Ahmed AR, Rickards G, Husain S, et al. Trends in internal hernia incidence after laparoscopic Roux-en-Y gastric bypass. Obes Surg. 2007;17(12):1563-6.

40. Madan AK, Lo Menzo E, Dhawan N, et al. Internal hernias and nonclosure of mesenteric defects during laparoscopic Roux-en-Y gastric bypass. Obes Surg. 2009;19(5):549-552.

41. Nguyen NT, Silver M, Robinson M, et al. Result of a national audit of bariatric surgery performed at academic centers: a 2004 University HealthSystem Consortium Benchmarking Project. Arch Surg. 2006;141(5):445-9. discussion 449-50.

42. Nguyen NT, Paya M, Stevens CM, et al. The relationship between hospital volume and outcome in bariatric surgery at academic medical centers. Ann Surg. 2004;240(4):586-93. discussion 593-4.

43. McCarty TM, Arnold DT, Lamont JP, et al. Optimizing outcomes in bariatric surgery: outpatient laparoscopic gastric bypass. Ann Surg. 2005;242(4):494-8. discussion 498-501.

44. Baker MT, Lara MD, Larson CJ, et al. Length of stay and impact on readmission rates after laparoscopic gastric bypass. Surg Obes Relat Dis. 2006;2(4):435-9.

45. Santry HP, Gillen DL, Lauderdale DS. Trends in bariatric surgical procedures. JAMA. 2005;294(15):1909-17. 